\title{
Resistant Hypertension and Atrial Fibrillation: How Important Is the Rhythm?
}

\section{Ebru İpek Türkoğlu1, ๑ Emine Çiğdem Kırçiçeği Çiçekdağ2, ๑ Eser Şekercioğlu3}

1İzmir Kemalpaşa State Hospital, Clinic of Cardiology, İzmir, Turkey

2İzmir Bornova Türkan Özilhan State Hospital, Clinic of Anesthesiology, İzmir, Turkey

32Dataist Analytics, Dublin, Ireland

\begin{abstract}
Objectives: Resistant hypertension (RHT) identifies a high-risk population; however, the effect of concomitant atrial fibrillation (AF) in this patient group is understudied. The present study aimed to investigate the effect of heart rhythm (HR) in this population.

Materials and Methods: The study was designed as a retrospective, single-center, closed cohort archive screening study in a given time and outpatient data were reviewed. Patients with secondary hypertension, pseudo-RHT and heart failure were eliminated. Consecutive 101 RHT patients were included in the study and divided into two groups according to HR, sinus rhythm (SR) or AF. Baseline demographics were compared, and the correlation between N-terminal pro-brain natriuretic peptide (NT-proBNP), left atrial diameter (LAD) and age were investigated.

Results: When comparing RHT patients according to HR, AF patients were older (mean age: $73.5 \pm 7.6$ vs. $61.7 \pm 11.2$ years, $\mathrm{p}<0.001)$, LAD was larger $(50.0 \pm 3.5$ vs. $40.9 \pm 4.0 \mathrm{~mm}, \mathrm{p}<0.001)$, LVEF was lower $(53.5 \pm 2.7$ vs. $57.3 \pm 2.8 \%$, $\mathrm{p}<0.001)$ and they had higher levels of NT-proBNP $(1704.5 \pm 868.9$ vs. $330.3 \pm 394.9 \mathrm{pg} / \mathrm{mL})$ than the RHT patients in SR. The correlation between LAD and NT-proBNP level was found only in RHT patients with SR, not with AF.
\end{abstract}

Address for Correspondence: Ebru İpek Türkoğlu, İzmir Kemalpaşa State Hospital, Clinic of Cardiology, İzmir, Turkey e-mail: dripek73@yahoo.com ORCID: orcid.org/0000-0002-2321-8868

Received: 01.09.2021 Accepted: 26.11.2021

Cite this article as: Türkoğlu Eİ, Kırçiçeği Çiçekdağ EÇ, Şekercioğlu E. Resistant Hypertension and Atrial Fibrillation: How Important Is the Rhythm? EJCM 2021;9(4):178-183.

DOI: 10.32596/ejcm.galenos.2021-09-046

Presented in: The present study had been presented orally in the $18^{\text {th }}$ National e-Congress of Hypertension and Cardiovascular diseases (18. Ulusal Hipertansiyon ve Kardiovasküler Hastalıklar e-Kongresi) on December 14-16, 2020. 
Conclusion: The co-presence of AF in RHT may indicate an advanced stage of the disease, not a simple arrhythmia, and the patients should be treated accordingly. Likewise, present study's findings indicate the need to specify the cut-off levels of NT-proBNP in AF in the absence of heart failure.

Keywords: Resistant hypertension, heart rhythm, atrial fibrillation, NT-proBNP, left atrial diameter

\section{Introduction}

Resistant hypertension (RHT) is the lack of blood pressure control despite appropriate treatment in an adherent patient. Appropriate treatment includes minimum three antihypertensive drugs from different classes with proper dosage and one should be diuretic by the definition. For the diagnosis, secondary hypertension and the contributing factors for the treatment resistance such as sleep apnea syndrome or regular non-steroidal anti-inflammatory drug use should be excluded. Hence pseudo-RHT is not so rare mainly due to patient's nonadherence or improper blood pressure measurement, true RHT is relatively uncommon in clinical practice, with an approximate estimation of 5\% of hypertensive patients, and identifies high-risk group ${ }^{(1,2)}$. Pathophysiology of RHT has not been fully solved yet; however, the effect of volume overload and aldosterone excess is already known ${ }^{(3)}$. Aging may also play an important role considering the higher prevalence of RHT in older patients ${ }^{(4-6)}$. Dilatation of left atrium (LA) is not uncommon in echocardiographic evaluation of hypertensive patients and reflects an impairment of the left ventricle diastolic functions. An important consequence of LA enlargement is the escalated atrial fibrillation (AF) risk. One of the important findings of the Framingham Study is that a five mm enlargement of LA detected by echocardiography raises AF incidence to $39 \%{ }^{(7,8)}$. The relationships between AF, stroke, mortality and morbidity are well known and the vigilance is increased in the last decade. Hence the volume overload is thought very important in the RHT, several studies investigated the role of natriuretic peptides (NP), which are secreted from the heart as a result of increased pressure and volume. Two main natriuretic peptides are in clinical use; B-type and N-terminal B-type natriuretic peptide (BNP and NTproBNP) and both give important prognostic information in multiple clinical scenarios beyond their role in volume homeostasis ${ }^{(9)}$. Although echocardiographic parameters, natriuretic peptides and co-morbid situation in RHT have been investigated previously, the interaction of heart rhythm with RHT is still understudied. With the aging of population and growing number of AF-patients, the topic becomes more popular. The present study aimed to compare RHT patients according to their heart rhythm and investigate the interaction of AF and RHT.

\section{Materials and Methods}

The outpatient data of our hypertension clinic were screened for the first 9 months of year 2015. Hence the clinic serves as the single second stage healthcare provider in a closed area with more than 100,000 residents, the data are considered as closed cohort data. The hospital communication system (HCS) records, which provided data about admission features such as initial complaint, personal history, blood tests, imaging results and medications of patients, were used. In the given nine months, data of 1,254 outpatients were reviewed retrospectively through the HCS. Inclusion criteria for the present study were either the uncontrolled blood pressure despite appropriate treatment with at least three drugs at best tolerated doses including a diuretic or requirement of four or more drugs to reach target blood pressure in treatment-adherent patient with the definitive diagnosis of hypertension. Adherence was monitored through controlling the refill of prescriptions via the social health security system. The exclusion criteria were the presence of secondary hypertension or white-coat hypertension, heart failure with reduced or preserved 
left ventricle ejection fraction (LVEF), and moderate to severe valvular disease, which may provoke arrhythmia and renal impairment (serum creatinine $>1.5 \mathrm{mg} / \mathrm{dL}$ ) or an estimated glomerular filtration rate (eGFR, calculated by the CKD-epi method) $<60 / \mathrm{mL} / \mathrm{min}$, which defines Grade 3a-4 renal insufficiency. After the inclusion and exclusion criteria were applied, 101 consecutive patients with RHT were identified fit for the analysis. The data about patient demographics (age, sex, complaints at application, blood pressure), laboratory and imaging study results were collected via hospital communication system. Prescriptions and compliance for treatment were checked through the online medication link of the national social security system.

NT-proBNP was the NP of choice, because of its more stable profile, and measurements were made with the Elecsys proBNP II assay/Cobas ${ }^{\circledR}$ system from Roche Diagnostics. With the knowledge of remarkable effect of age and gender on NT-proBNP, age and gender specific upper limits of $97.5^{\text {th }}$ percentile for NT-proBNP were used, as defined in the prospectus information of the Elecsys proBNP II assay.

In the statistical analysis, mean \pm standard deviation and percentages were given for continuous and categorical variables respectively. All the analyses were performed with the IBM SPSS-22 software. The Pearson's correlation test was the test performed to investigate the relationships between variables. Statistical significance was considered as $\mathrm{p}<0.05$.

As the study was planned as a retrospective survey, no informed consent was obtained. Both of the authors had no conflict of interest and no external funding was received for the study. The number of ethical approval for the study was 16-10.1/3 and it was obtained from the Clinical Research Ethics Board of Ege University School of Medicine on 22.11.2016.

\section{Results}

After applying all the inclusion and exclusion criteria to 1,254 patients through HCS in the first 9 months of 2015,
101 consecutive patients were identified as true RHT, with an incidence of $8.05 \%$. The most frequent complaints on application reports were exercise intolerance and fatigue. Among 101 patients, 31 (30.7\%) were male and 70 were female with a mean age of $64.9 \pm 11.6$ years. Appropriate doses of either angiotensin-converting enzyme inhibitor or angiotensin receptor blocker plus diuretic were included in the prescription of all patients. Beta-blockers in 30 patients and calcium antagonists in 26 patients were third drug of choice. Forty-five patients needed four or more drugs. Twenty-seven patients were type 2 diabetic and none of them received any glitazone treatment. Only three patients were on intensive insulin treatment. The mean blood pressure was $170.4 \pm 19.8 \mathrm{mmHg}$ systolic and $101.8 \pm 91.2 \mathrm{mmHg}$ diastolic. The electrocardiographic and echocardiographic reports were obtained from HCS. Left ventricle hypertrophy $(\mathrm{LVH})$ and diastolic dysfunction were present in all of RHT patients. The mean LVEF was $56.3 \pm 3.3 \%$ and the mean LAD was $43.4 \pm 5.6 \mathrm{~mm}$. The mean creatinine was $1.0 \pm 0.2 \mathrm{mg} / \mathrm{dL}$ and NT-proBNP $697.6 \pm 826.7 \mathrm{pg} / \mathrm{mL}$ in the study group. Descriptive demographics of the group are given in Table 1.

After the definition of the basal demographics of the study population, RHT patients were grouped based on sinus rhythm (SR) or AF. The numbers of RHT patients in the AF-group and in the SR-group were 27 and 74, respectively. The AF group was older (73.5 \pm 7.6 vs. $61.7 \pm 11.2$ years, $\mathrm{p}<0.001)$, with a higher heart rate $(85.3 \pm 16.8$ vs. $72.7 \pm 11.8$ bpm, $\mathrm{p}<0.001)$, larger LAD $(50.0 \pm 3.5$ vs. $40.9 \pm 4.0 \mathrm{~mm}, \mathrm{p}<0.001)$, lower $\mathrm{LVEF}$ $(53.5 \pm 2.7$ vs. $57.3 \pm 2.8 \%, \mathrm{p}<0.001)$ and higher NTproBNP level $(1704.5 \pm 868.9$ vs. $330.3 \pm 394.9 \mathrm{pg} / \mathrm{mL}$, $\mathrm{p}<.001)$ than the SR-group. Both groups had similar blood pressure levels. Although creatinine was higher in $\mathrm{AF}$-patients $(1.1 \pm 0.2$ vs. $0.96 \pm 0.2 \mathrm{mg} / \mathrm{dL}, \mathrm{p}=0.004)$, other blood results remained alike in both groups. Hence all the eGFR results were defined as $>60 \mathrm{ml} / \mathrm{min}$ (absence of renal failure), a comparison between the groups were not made. Antihypertensive medication was similar in both groups (all the patients with AF and SR did received appropriate dosage of a RAS blocker, either ACEİ or ARB with diuretic); however, AF patients tended to receive 
Table 1. Group demographics

\begin{tabular}{|l|l|}
\hline & RHT patients $(\mathbf{n = 1 0 1})$ \\
\hline Gender & Male: $30.7 \%(\mathrm{n}=31)$ \\
\hline Age (years) & Female: $69.3 \%(\mathrm{n}=70)$ \\
\hline HR (bpm) & $64.91 \pm 11.63$ \\
\hline SBP (mmHg) & $76.11 \pm 14.43$ \\
\hline DBP (mmHg) & $170.48 \pm 19.85$ \\
\hline NT-proBNP (pg/mL) & $101.83 \pm 91.26$ \\
\hline LAD (mm) & $697.68 \pm 826.73$ \\
\hline LVEF (\%) & $43.40 \pm 5.65$ \\
\hline Ascendan aorta diameter (mm) & $56.34 \pm 3.31$ \\
\hline Fasting glucose (mg/dL) & $35.48 \pm 3.66$ \\
\hline Urea (mg/dL) & $119.66 \pm 41.42$ \\
\hline Creatinine (mg/dL) & $36.91 \pm 13.00$ \\
\hline Potassium (mmol/L) & $1.00 \pm 0.23$ \\
\hline LDL (mg/dL) & $4.40 \pm 0.46$ \\
\hline TG (mg/dL) & $112.27 \pm 35.61$ \\
\hline CRP (mg/dL) & $158.45 \pm 72.21$ \\
\hline$R H T: R e s i a n$ \\
\hline
\end{tabular}

RHT: Resistant hypertension, HR: Heart rate, SBP: Systolic blood pressure, DBP: Diastolic blood pressure, LAD: Left atrial diameter, LVEF: Left ventricle ejection fraction, LDL: Low-density lipoprotein, TG: Triglyceride, CRP: C-reactive protein, NT-proBNP: N-terminal pro-brain natriuretic peptide, $n$ : Number more b-blockers than the patients in SR (24/27 vs. 51/71 patients, $\mathrm{p}=0.038$ ). Comparison of two groups is given in Table 2.

In the third part, the relationship between NT-proBNP and some clinical variables was investigated in both groups. In the SR-group, NT-proBNP and left atrial diameter (LAD) were correlated (pairwise $\mathrm{r}=0.451$, $\mathrm{p}<0.001$ ), and the correlation continued even partially $(r=0.234, p<0.049)$ after being controlled for variables known or thought to be related to NT-proBNP such as age, sex, heart rate, systolic and diastolic blood pressures. On the contrary, in the AF-group, no correlation was found between NT-proBNP and LAD, neither pairwise nor partial (respectively $\mathrm{r}=0.208, \mathrm{p}=0.297$ and $\mathrm{r}=0.330$, $\mathrm{p}=0.134$, after controlling same variables as age, sex, HR, SBP and DBP). Similar relationships were obtained while searching the correlations between NT-proBNP and age in both groups. In RHT patients with SR, there was a significant partial (after controlling for LAD, gender, HR, SBP and DBP) as well as pairwise correlation between NT-proBNP and age, which looked significant only in pairwise analysis. After controlling the variables, the

Table 2. Two groups are defined according to the heart rhythm in the RHT population and the comparison of two groups

\begin{tabular}{|c|c|c|c|}
\hline Rhythm & AF $(n=27)$ & $S R(n=74)$ & p-value \\
\hline \multirow{2}{*}{ Gender } & M (5) $18.5 \%$ & (26) $35.1 \%$ & \multirow{2}{*}{0.109} \\
\hline & $F(22) 81.5 \%$ & (48) $64.9 \%$ & \\
\hline Age (years) & $73.56 \pm 7.62$ & $61.76 \pm 11.27$ & $<0.001$ \\
\hline HR (bpm) & $85.33 \pm 16.88$ & $72.74 \pm 11.87$ & $<0.001$ \\
\hline SBP $(\mathrm{mmHg})$ & $164.78 \pm 17.98$ & $172.55 \pm 20.21$ & 0.081 \\
\hline $\mathrm{DBP}(\mathrm{mmHg})$ & $125.19 \pm 175.31$ & $93.31 \pm 13.74$ & 0.121 \\
\hline NT-proBNP (pg/mL) & $1,704.59 \pm 868.19$ & $330.30 \pm 394.96$ & $<0.001$ \\
\hline LAD (mm) & $50.07 \pm 3.55$ & $40.96 \pm 4.09$ & $<0.001$ \\
\hline LVEF (\%) & $53.52 \pm 2.71$ & $57.36 \pm 2.89$ & $<0.001$ \\
\hline Ascendan aorta diameter $(\mathrm{mm})$ & $35.67 \pm 3.54$ & $35.41 \pm 3.73$ & 0.753 \\
\hline Fasting glucose (mg/dL) & $117.89 \pm 32.88$ & $120.31 \pm 44.31$ & 0.796 \\
\hline Urea (mg/dL) & $40.67 \pm 12.19$ & $35.54 \pm 13.10$ & 0.079 \\
\hline Creatinine (mg/dL) & $1.10 \pm 0.25$ & $0.96 \pm 0.21$ & 0.004 \\
\hline Potassium (mmol/L) & $4.54 \pm 0.46$ & $4.34 \pm 0.46$ & 0.055 \\
\hline LDL (mg/dL) & $106.38 \pm 31.49$ & $114.37 \pm 36.94$ & 0.328 \\
\hline TG (mg/dL) & $140.22 \pm 74.08$ & $165.19 \pm 70.84$ & 0.125 \\
\hline CRP (mg/dL) & $4.61 \pm 6.50$ & $5.06 \pm 4.91$ & 0.775 \\
\hline
\end{tabular}

The values are given as mean \pm standard deviation. Significant $p$-values are shown in bold.

RHT: Resistant hypertension, HR: Heart rate, AF: atrial fibrillation, SR: sinus rhythm, M: Male, F: Female, SBP: Systolic blood pressure, DBP: Diastolic blood pressure, NT-proBNP: N-terminal pro-brain natriuretic peptide, LAD: Left atrial diameter, LVEF: Left ventricle ejection fraction, LDL: Low-density lipoprotein, TG: Triglyceride, CRP: C-reactive protein, n: Number 
partial correlation between NT-proBNP and age became not significant. In contrary, the AF-group represented not any significant relationship between NT-proBNP, age or LAD. Results are given in Table 3.

\section{Discussion}

Hypertension (HT) and AF are two important problems of daily clinical practice and they often coexist ${ }^{(10)}$. While the Framingham data suggest that hypertension might foretell $\mathrm{AF}$, the 15-year follow-up data of the study indicate that more effective $\mathrm{BP}$ control does not reduce $\mathrm{AF}$ occurrence in the hypertensive patients ${ }^{(8,11)}$. Although the relationship between HT and AF is well established, the underlying mechanism is not been completely solved ${ }^{(10)}$. Aldosterone excess, HT and AF may be related in multiple ways. The activation of renin-angiotensin system increases aldosterone synthesis and increased aldosterone levels are associated with $\mathrm{HT}, \mathrm{AF}, \mathrm{LVH}$, and diastolic dysfunction in general population ${ }^{(12)}$. Aldosterone endorses myocardial remodeling and AF. Hence AF develops, it further increases aldosterone level creating a vicious circle ${ }^{(13)}$. Whether the AF-risk increases linearly with BP or there is a BP threshold, above which the risk increases, is not known ${ }^{(10)}$. Furthermore, the blood pressure goal in this population is also uncertain although available data do not recommend lowering $\mathrm{BP}$ in $\mathrm{AF}$ patients below current targets $^{(10,12)}$.
HT is a modifiable cardiovascular risk factor, but treatment resistance is a real problem. Prevalence studies report the treatment resistance as 5\%-30\% in the hypertensive patients, which includes pseudo-resistant and treatment non-compliant patients. After applying strict definition criteria, the rate of RHT is thought to be under $10 \%$ of all patients with hypertension. RHT defines an important high-risk population for target organ injury, long-term renal disease and premature cardiovascular events mediated by hypertension ${ }^{(1)}$. The definition of resistant hypertension is basically a rule-out diagnosis. The patient, whose blood pressure is not controlled, should be adherent to treatment, the treatment should contain a combination of proper drugs in appropriate dosages, and all of the causes of secondary hypertension and treatment resistance should be excluded ${ }^{(1)}$. However, this kind of definition may put different diseases with various etiologies and underlying pathologies in the same diagnostic basket, which could lead the inappropriate treatment of individuals with similar appearance but different underlying pathophysiological mechanism.

The mechanism, which is responsible for RHT, may not be a simple single one and perhaps underlying pathophysiological causes may differ by age; however, Gaddam et al. ${ }^{(3)}$ described one of them as the aldosterone excess with an increased intravascular volume. They demonstrated higher levels of NP's in RHT compared to

Table 3. The correlation between NT-proBNP, left atrial diameter and age in both groups according to the heart rhythm in RHT

\begin{tabular}{|c|c|c|c|c|c|}
\hline & & & LAD & & \\
\hline & & SR & AF & SR & AF \\
\hline & Pairwise & $0.451(p<0.001)$ & $0.208(p=0.297)$ & $0.670(p<0.001)$ & $\begin{array}{l}0.173 \\
(p=0.387)\end{array}$ \\
\hline NT-proBNP & Partial & $0.234(p=0.049)^{a}$ & $0.330,(p=0.134)^{a}$ & $0.590(p<0.001)^{b}$ & $\begin{array}{l}0.183 \\
(p=0.415)^{b}\end{array}$ \\
\hline & Pairwise & - & - & $0.469(p<0.001)$ & $\begin{array}{l}-0.142 \\
(p<0.001)\end{array}$ \\
\hline LAD & Partial & - & - & $0.175(p=0.151)^{c}$ & $\begin{array}{l}-0.246 \\
(p=0.269)^{c}\end{array}$ \\
\hline
\end{tabular}


controls, which was more pronounced in male gender ${ }^{(3)}$. The correlation between NPs and adverse cardiac outcomes is well known, and because of that, NPs are utilized in many clinical scenarios to identify high-risk patients beyond their role in volume homeostasis ${ }^{(9)}$. Defining the upper limits of normal is also not so simple for NT-proBNP considering the important impact of age and gender. Previously underlined $125 \mathrm{pg} / \mathrm{ml}$ level of NT-proBNP is basically a rule-out level for acute heart failure derived mainly from the PRIDE-study and it may be improper to use a rule-out level for any rule-in process $^{(14,15)}$. New European NP guideline has changed the rule-in levels for heart failure in accord with FDA recommendations; however, it may also be improper to use any heart failure rule-in level for the diagnosis of hypervolemia $^{(16)}$. A former paper of us on RHT, which used gender and age specific cut-offs for NT-proBNP in the $97.5^{\text {th }}$ percentile, suggested that high levels of NT-proBNP did not exist in every RHT patients. The study suggested only the elderly patients with RHT had higher levels of NT-proBNP, which may indicate high risk of adverse events as well as hypervolemia ${ }^{(17)}$. Considering not all but elderly RHT patients have higher levels of NT-proBNP, a speculation can be made that these patients might be at greater risk for AF because of volume overload and higher risk, which is assigned by elevated levels of NT-proBNP and age. Identifying the high-risk patients is crucial for an accurate approach. Although RHT population is studied widely, the impact of rhythm in this population is not well described. As the number of patients with $\mathrm{AF}$ is growing each day and AF burden becomes a real clinical issue, as a subgroup, RHT patients with AF are not defined decently. The present study investigated RHT patients of a closed cohort and compared the features of these patients according to their rhythms. RHT patients with AF were older, which is consistent with the knowledge of increasing prevalence of AF with aging ${ }^{(18)}$. This finding also might indicate that the development of AF in RHT patients addresses the disease advance in years; however, whether all the patients would develop AF with aging or which patients would develop AF in years and what could be done to prevent it are not completely known yet. In the present study group, RHT patients in AF had higher HR, NT-proBNP and creatinine levels, larger LAD and lower LVEF than RHT patients in SR, which might be related to a more advanced phase of the disease. Higher creatinine levels, which may indicate deteriorating of renal function, larger LAD and lower LVEF can be attributed directly to the AF, because the interaction between $\mathrm{AF}$ and renal function is well known as the cardiac function ${ }^{(18)}$. Hence the relationship of AF and NT-proBNP is recognized well for years, the higher level of that biomarker in atrial fibrillation group has been found not to be surprising. Several studies have suggested that the NT-proBNP levels of $800-1100 \mathrm{pg} / \mathrm{mL}$ should be accepted as a median in patients with atrial fibrillation and preserved left ventricle ejection fraction. Not any correlation of biomarker with duration of AF or LAD is found ${ }^{(19)}$. Uncontrolled blood pressures may lead AF, and also as a marker, high plasma levels of NT-proBNP can predict future onset of AF independently ${ }^{(18,20)}$. Uncontrolled blood pressure over years and also increased NT-proBNP levels in resistant hypertensive patients may explain high percentage of AF patients in RHT group. It may give rise to the thought that older RHT patients having increased levels of NT-proBNP would develop AF over years if the blood pressure could not be controlled well and required precautions are not taken. The present study found a relationship between NTproBNP, LAD and age, in resistant hypertensive patients only on sinus rhythm but not on atrial fibrillation. The lack of correlation in the AF-group may be due to that all patients have higher levels of that biomarker and larger LAD. This finding may mean that current cutoffs for NTproBNP may not be appropriate for patients with $\mathrm{AF}$, and AF-specific cutoffs are needed for these patients.

\section{Study Limitations}

The most important limitation of the study is its retrospective design. Single center design is another limitation, which led to limited number of patients, although the data of a closed cohort were used. Using ambulatory blood pressure holter instead of the office- 
based BP levels could be much better even the blood pressures were measured according to the guidelines by a hypertension-nurse well educated in this area. The prescription information and treatment adherence of patients were checked via online social security system, but it is always possible that patients did not take the pills regularly even the pills were refilled. Despite these limitations, the study presents real-world clinical data and compares RHT patients according to their rhythm, while questioning the effect of rhythm in RHT population, which represents a growing and understudied part of hypertensive patients.

\section{Conclusion}

Resistant hypertension, elevated levels of NT-proBNP and atrial fibrillation are important parts of a daily clinical dilemma, where age plays a fundamental role. In our aging society, with the longer expectation of survival, the borders between cardiovascular diseases become hazy. The present study suggests that uncontrolled BP and high NT-proBNP levels may lead or foretell AF development in RHT patients over years, and the elderly RHT patients with elevated levels of NT-proBNP should be monitored closely for the development of AF and every precaution should be taken to prevent it. Once AF develops, these patients differ from the patients in SR, which might be speculated as transition to a more advanced phase of the disease or another disease. The positive correlation between NT-proBNP and LAD is found only in RHT patients with SR, but not with AF, which may indicate the need for specific cut-off levels of NT-proBNP in AF.

\section{Ethics}

Ethics Committee Approval: This study was approved by the Clinical Research Ethics Board of Ege University School of Medicine on 22.11.2016 (no: 16-10.1/3).

Informed Consent: Retrospective study.

Peer-review: Externally peer-reviewed.

\section{Authorship Contributions}

Conception and Design: E.İ.T., E.Ç.K.Ç., E.Ş., Acquisition of Data: E.İ.T., E.Ç.K.Ç., Analysis and/ or Interpretation of Data: E.İ.T., E.Ş., Drafting of the Manuscript: E.İT., Critical Revisions: E.İ.T., E.Ç.K.Ç., E.Ş., Statistical Analysis: E.Ş., Technical and Material Support: E.İ.T., E.Ç.K.Ç., Supervision: E.İ.T.

Conflict of Interest: The authors declare that they had no conflict of interest.

Financial Disclosure: The authors declare no financial support by any grant or research sponsor and no competing financial interest.

\section{References}

1. Williams B, Mancia G, Spiering W, et al. The task force for the management of arterial hypertension of the European Society of Cardiology (ESC) and the European Society of Hypertension (ESH). 2018 ESC/ESH Guidelines for the management of arterial hypertension. Eur Heart J 2018;39:3021104.

2. Townsend RR, Epstein M. Resistant Hypertension. Insights on evaluation and management in the post-SPRINT (systolic blood pressure intervention trial) era. Hypertension 2016;68:1073-80 .

3. Gaddam KK, Nishizaka MK, Pratt-Ubunama MN, et al. Resistant hypertension characterized by increased aldosterone levels and persistent intravascular volume expansion. Arch Intern Med 2008;168:1159-64.

4. Sarafidis PA, Bakris GL. Resistant hypertension an overview of evaluation and treatment. J Am Coll Cardiol 2008;52:1749-57.

5. Sarafidis PA, Bakris GL. State of hypertension management in the United States: confluence of risk factors and the prevalence of resistant hypertension. J Clin Hypertens (Greenwich) 2008;10:130-9.

6. Calhoun DA, Zaman MA, Nishizaka MK. Resistant hypertension. Curr Hypertens Rep 2002;4:221-8.

7. Chen Y, Sato H, Watanabe N, et al. Factors influencing left atrial volume in treated hypertension. J Cardiol 2012;60:133-8.

8. Benjamin EJ, Levy D, Vaziri SM, D’Agostino RB, Belanger AJ, Wolf PA. Independent risk factors for atrial fibrillation in a population-based cohort. The Framingham Heart Study. JAMA 1994;271:840-4.

9. Daniels LB, Maisel AS. Natriuretic peptides. J Am Coll Cardiol 2007;50:2357-68

10. Verdecchia P, Angeli F, Reboldi G. Hypertension and atrial fibrillation. Doubts and certainties from basic and clinical studies. Circ Res 2018;122:352-68.

11. Rahman F, Yin X, Larson MG, et al. Trajectories of risk factors and risk of new-onset atrial fibrillation in the Framingham Heart Study. Hypertension 2016;68:597-605. 
12. Dzeshka MS, Shantsila A, Shantsila E, Lip GYH. Atrial fibrillation and hypertension. Hypertension 2017;70:854-64.

13. Seccia TM, Caroccia B Adler GK, Maiolino G, Cesari M, Rossi GP. Arterial hypertension, atrial fibrillation, and hyperaldosteronism: the triple trouble. Hypertension 2017;69:545-50.

14. Januzzi Jr JL, Camargo CA, Anwaruddin S, et al. The N-terminal Pro-BNP investigation of dyspnea in the emergency department (PRIDE) study. Am J Cardiol 2005;95:958-54.

15. Ponikowski P, Voors AA, Anker SD, et al. 2016 ESC Guidelines for the diagnosis and treatment of acute and chronic heart failure. Eur Heart $\mathrm{J}$ 2016;37:2129-200

16. Mueller C, McDonald K, de Boer RA, et al; on behalf of the Heart Failure Association of the European Society of Cardiology. Heart Failure Association of the European Society of Cardiology practical guidance on the use of natriuretic peptide concentrations Eur J Heart Fail 2019;21:71531.
17. Turkoglu EI, Kircicegi Cicekdag EC. Resistant hypertension in elderly: a clinical manifestation of heart failure with preserved ejection fraction? Retrospective single-center analysis. Clin Exp Hypertens 2019;41:505-10.

18. Kirchof P, Benussi S, Kotecha D, et al. The task force for the management of atrial fibrillation of the European Society of Cardiology, developed with the special contribution of the European Heart Rhythm Association (EHRA) of the ESC, endorsed by the European Stroke Organisation (ESO). 2016 ESC Guidelines for the management of atrial fibrillation developed in collaboration with EACTS. Eur Heart J 2016;37:2893-966.

19. Marsiliani D, Bucceletti F, Carroccia A, Gilardi E, Silveri NG, Franceschi F. Natriuretic peptides and atrial fibrillation. Eur Rev Med Pharmacol Sci 2010:14:855-60

20. Asselbergs FW, Van Den Berg MP, Bakker SJ, et al. N-terminal pro B-type natriuretic peptide levels predict newly detected atrial fibrillation in a population-based cohort. Neth Heart J 2008;16:73-8. 